\title{
Ultra-high degree spherical harmonic analysis and synthesis using extended-range arithmetic
}

\author{
Tobias Wittwer • Roland Klees • Kurt Seitz • \\ Bernhard Heck
}

Received: 31 October 2006 / Accepted: 2 June 2007 / Published online: 12 July 2007

(C) Springer-Verlag 2007

\begin{abstract}
We present software for spherical harmonic analysis (SHA) and spherical harmonic synthesis (SHS), which can be used for essentially arbitrary degrees and all co-latitudes in the interval $\left(0^{\circ}, 180^{\circ}\right)$. The routines use extended-range floating-point arithmetic, in particular for the computation of the associated Legendre functions. The price to be paid is an increased computation time; for degree 3,000 , the extended-range arithmetic SHS program takes 49 times longer than its standard arithmetic counterpart. The extended-range SHS and SHA routines allow us to test existing routines for SHA and SHS. A comparison with the publicly available SHS routine GEOGFG18 by Wenzel and HARMONIC SYNTH by Holmes and Pavlis confirms what is known about the stability of these programs. GEOGFG18 gives errors $<1 \mathrm{~mm}$ for latitudes $\left[-89^{\circ} 57.5^{\prime}, 89^{\circ} 57.5^{\prime}\right]$ and maximum degree 1,800 . Higher degrees significantly limit the range of acceptable latitudes for a given accuracy. HARMONIC SYNTH gives good results up to degree 2, 700
\end{abstract}

\footnotetext{
T. Wittwer $(\varangle) \cdot$ R. Klees

Delft Institute of Earth Observation and Space Systems (DEOS),

Delft University of Technology, Kluyverweg 1,

2629 HS Delft, The Netherlands

e-mail: t.f.wittwer@tudelft.nl

R. Klees

e-mail: R.Klees@tudelft.nl

K. Seitz · B. Heck

Geodetic Institute, University of Karlsruhe,

Englerstr. 7, 76128 Karlsruhe, Germany

e-mail: seitz@gik.uni-karlsruhe.de

B. Heck

e-mail: heck@gik.uni-karlsruhe.de
}

for almost the whole latitude range. The errors increase towards the North pole and exceed $1 \mathrm{~mm}$ at latitude $82^{\circ}$ for degree 2, 700. For a maximum degree 3, 000, HARMONIC SYNTH produces errors exceeding $1 \mathrm{~mm}$ at latitudes of about $60^{\circ}$, whereas $G E O G F G 18$ is limited to latitudes below $45^{\circ}$. Further extending the latitudinal band towards the poles may produce errors of several metres for both programs. A SHA of a uniform random signal on the sphere shows significant errors beyond degree 1, 700 for the SHA program SHA by Heck and Seitz.

Keywords Spherical harmonic analysis $\cdot$ Spherical harmonic synthesis - Extended-range arithmetic . Numerical stability

\section{Introduction}

Spherical harmonic analysis (SHA) and spherical harmonic synthesis (SHS) are basic tools in Earth sciences. The wealth of data provided by space-borne, airborne and terrestrial measurement sensors allows the computation of spherical harmonic expansions far beyond degree, say, 360. Today, expansions of degree 2,160 or higher have become feasible in real data processing or simulations (e.g., Pavlis et al. 2005). It is expected that the degree of spherical harmonic models will continue to increase, although combinations of moderate-degree spherical harmonic expansions with spherical basis function representations (e.g., wavelets) are becoming more popular (e.g., Freeden and Windheuser 1997).

When ultra-high degree $(2,000)$ spherical harmonic expansions are used for data analysis and modelling, three basic numerical aspects become important: (i) the numerical efficiency of the algorithm, (ii) the stability of the recurrence relations that are used to compute the associated Legendre 


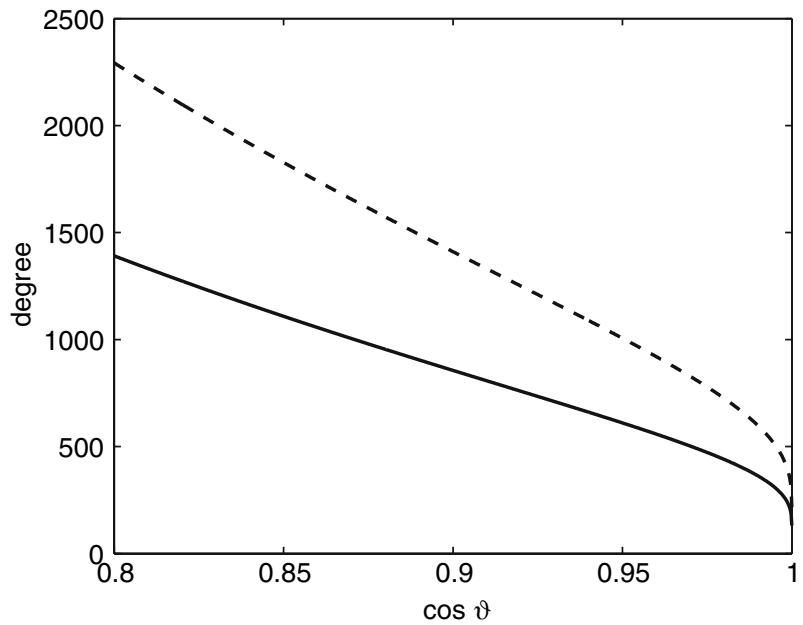

Fig. 1 Maximum admissible spherical harmonic degree $n$ for IEEE double precision as a function of $\cos \vartheta$. Solid line without re-scaling. Dashed line re-scaling with factor $10^{200}$ as proposed by Wenzel (1998) and implemented in GEOGFG18

functions (ALFs), and (iii) the underflow of early values in the recurrence relations.

Much literature has been devoted to the numerical efficiency of algorithms and to the stability of recurrence relations, in particular in geodesy, meteorology, and numerical mathematics (Tscherning and Poder 1982; Tscherning et al. 1983; Gleason 1985; Koop and Stelpstra 1989; Driscoll and Healy 1994; Holmes 2003).

Much less attention has been paid to the problem of underflow. This problem is easily visible in the asymptotic approximation (Smith et al. 1981)

$\bar{P}_{n, n} \sim \frac{1}{2}\left(\frac{n}{\pi}\right)^{1 / 4}\left(1-t^{2}\right)^{n / 2}, \quad n \rightarrow \infty, t$ fixed,

where $\bar{P}_{n, n}$ is the fully normalized ALF of degree and order $n$, $t=\cos \vartheta$, and $\vartheta$ is the spherical co-latitude. From Eq. (1), we can determine the maximum admissible degree $n$ for a given $t$ as function of the smallest non-zero positive and the largest non-zero negative normalized number $\omega$ that is storable for the given compiler and software:

$n_{\max }<\frac{2 \alpha-\frac{1}{2} \lg \frac{2 \alpha}{\pi x}}{x} \approx \frac{2 \alpha}{x}$,

where $\alpha=\lg 2 \omega$ and $x=\lg \left(1-t^{2}\right)$.

According to the IEEE standard for binary floating-point arithmetic in double precision (which applies to a Pentium IV processor), it is $\omega \approx \pm 2.225 \times 10^{-308}$. For degrees $n>n_{\max }$, the standard recursion commonly used to compute $\bar{P}_{n, m}$ cannot be initialized due to underflow during the computation of the seeds $\bar{P}_{n, n}$. Figure 1 shows $n_{\max }$ as function of the argument $t$ for $\omega$ according to the IEEE double precision standard.
For instance, underflow will occur in IEEE double precision for co-latitudes outside the interval $\left[21.7^{\circ}, 158.3^{\circ}\right]$ and $\left[45.9^{\circ}, 134.1^{\circ}\right]$ if the maximum degree is 720 and 2,160 , respectively.

The simplest way of alleviating the underflow problem would be to employ multiple-precision software (e.g., Brendt 1978). Koop and Stelpstra (1989) propose to scale the ALFs when an underflow is about to occur. Wenzel (1998) scales all the computations upwards by a factor of $10^{200}$. This extends the interval of admissible co-latitudes to $\left[11.3^{\circ}, 168.7^{\circ}\right]$ and $\left[35.5^{\circ}, 144.5^{\circ}\right]$ for a maximum degree 720 and 2,160 , respectively.

A more sophisticated treatment of the underflow problem is in Holmes and Featherstone (2002). They use a method proposed by Libbrecht (1985), which is based on recurrence relations for $\bar{P}_{n, m}(\cos \vartheta) / \sin ^{m}(\vartheta)$. In this way, the problematic $\sin ^{m} \vartheta$ term is eliminated from the recursive algorithms; later on, this term is gradually re-introduced employing Horner's scheme. To avoid overflow during the recursions, a scale factor of $10^{-280}$ is introduced. They conclude that spherical harmonic expansions up to degree 2,700 can be computed without either underflow or overflow according to the IEEE standard for binary floating-point arithmetic in double precision for all co-latitudes. No tests with 'real' spherical harmonic coefficients were presented, but are in Holmes (2003).

Jekeli et al. (2007) have proposed a new approach for the computation of ALFs. It is mainly based on the observation that ALFs show a very strong attenuation in the degree-andorder domain for specific orders as function of the degree and the co-latitude. The attenuation is used to neglect insignificant ALFs. This requires the use of other recursions than usually used, which are known to be unstable. Their simulations indicate that the errors can be made smaller than the noise level up to degrees of at least 10,800 at all latitudes.

The subject of the short note is a special form of computer floating-point arithmetic, which is introduced in Smith et al. (1981). The basic idea of this extended-range arithmetic is to allocate a separate storage location to the exponent of each floating-point number. As such, there is no need to use modified recursions or to introduce any re-scaling in the course of the computations.

In extended-range arithmetic, the smallest number, which can be represented in base ten, according to the IEEE standards, is $10^{-10343312045}$. Hence, ALFs up to ultra-high degree can be computed for all latitudes in the interval $\left(0^{\circ}, 180^{\circ}\right)$. Figure 2 shows $n_{\max }$ (Eq. (2)) as a function of $t$ in extendedrange arithmetic. For instance, suppose an expansion up to degree $n=100,000$. Let us assume that the smallest colatitude to be processed is $\frac{\pi}{100,000} \mathrm{rad}$. In standard doubleprecision arithmetic, the maximum degree $\bar{P}_{n, n}$ is $n_{\max }=68$. In extended range-arithmetic, $n_{\max } \approx 4.56 \times 10^{9}$. 


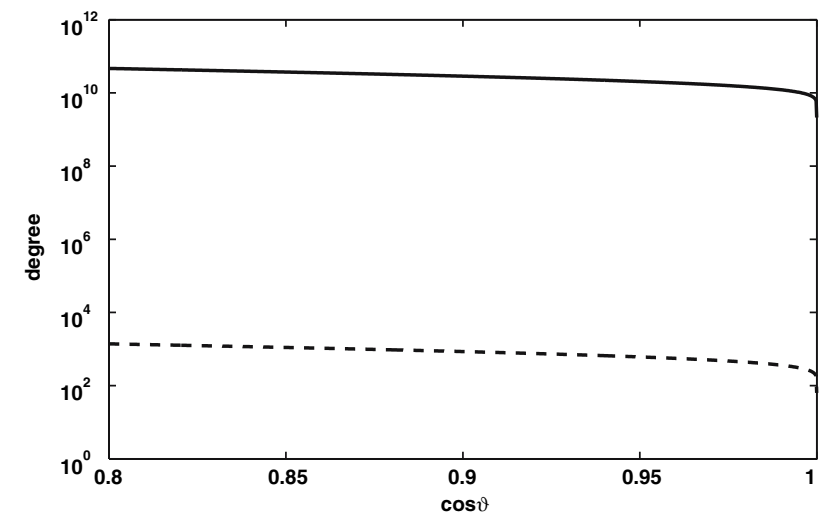

Fig. 2 Maximum admissible spherical harmonic degree $n$ for IEEE double precision (dashed line) and IEEE extended-range arithmetic (solid line) as a function of $\cos \vartheta$

When extended-range arithmetic is applied to the recursive computation of ALFs, there is a loss of precision, which does not exceed (Smith et al. 1981)

$\lg n+\lg (10+2|\vartheta \cot \vartheta|)$.

Equation (3) is shown in Fig. 3. For $\vartheta=179.982^{\circ}$, we loose about 10 digits for degree 10,000 ; for $\vartheta=179.9982^{\circ}$, we loose about 12 digits for degree 100, 000 .

Lozier and Smith (1981) developed an extended-range software package, which contains a number of useful routines, to support the development of our extended-range arithmetic SHA and SHS software. This package is now part of the SLATEC Common Mathematical Library, a software library containing over 1, 400 general purpose mathematical and statistical routines written in Fortran 77. The current version is 4.1, which was released in July 1993 (see http:// performance.netlib.org/slatec/).

The objective of the short note is twofold: (i) to investigate the numerical performance of extended-range arithmetic SHA and SHS programs, and to compare it with the performance of standard double precision arithmetic SHA and SHS programs. Smith et al. (1981) report that the extended-range arithmetic computation of the ALFs is a factor two slower than the corresponding double-precision computation. Nothing is known about whether this also applies to SHA and SHS; (ii) we want to investigate the numerical stability of publicly available SHA and SHS programs.

\section{Numerical tests}

Extended-range floating-point arithmetic SHA and SHS software has been developed, which makes use of routines of Lozier and Smith (1981). The SHA program SHA developed by Heck and Seitz (1991, 1993), and the SHS program GEOGFG18 of Wenzel (1998) form the basis of the

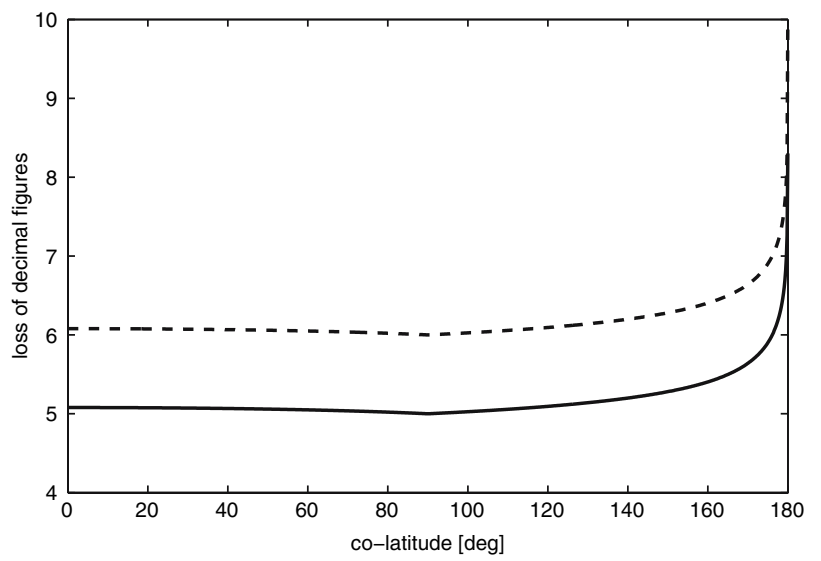

Fig. 3 Loss of significant decimal figures when computing $\bar{P}_{n, n}(t)$ with extended-range arithmetic as a function of the co-latitude. Solid line $n=100,000$, range $\left[0.0018^{\circ}, 179.9982^{\circ}\right]$. Dashed line $n=10,000$, range $\left[0.018^{\circ}, 179.982^{\circ}\right]$

new routines, which are called ERA-SHA and ERA-SHS, respectively.

The correctness of ERA-SHA and ERA-SHS was checked by synthesizing gravity potential values up to degree 1,800 on a global grid, using Wenzel's GPM98b potential model and $E R A-S H S$. These gravity potential values were then used to estimate the original potential coefficients using SHA with the program ERA-SHA. The resulting error degree variances were in the range of $10^{-15}$, the magnitude of numerical accuracy. Moreover, the computation of the ALFs were compared for various degrees and latitudes with the values published in Lozier and Smith (1981). Finally, we used relationships for the sum of ALFs for validation. Therefore, we safely assume both programs to work correctly up to degree 1,800 .

We performed a number of numerical tests to assess the influence of using extended-range arithmetic. All tests were performed on an Intel Pentium D 830 clocked at $3 \mathrm{GHz}$, using Intel's Fortran compiler 9.1 under Linux. All programs were run single-threaded in 64 bit-mode.

\section{Results}

The first series of tests was devised to quantify the impact of using extended-range arithmetic on computation times. For SHS, we used Wenzel's GEOGFG18 and ERA-SHS. Values were computed on a regular global grid for various maximum spherical harmonic degrees $n_{\max }$; the grid spacing was chosen according to $\Delta \vartheta=\Delta \lambda=\frac{\pi}{n_{\max }} \mathrm{rad}$.

Table 1 and Fig. 4 show the resulting run-times as function of $n_{\max }$. The extended-range arithmetic SHS requires significantly more computation time than standard arithmetic SHS. For instance, at $n_{\max }=1,800$, the computation time is a factor 40 larger. This is much above the factor of 
Table 1 SHS run-times and run-time ratios for standard and extended-range arithmetic at various maximum degrees $n_{\max }$

\begin{tabular}{llccr}
\hline$n_{\max }$ & Spacing & Standard $[\mathrm{s}]$ & Extended $[\mathrm{s}]$ & Ratio \\
\hline 30 & $6^{\circ}$ & 4.3 & 4.7 & 1.1 \\
60 & $3^{\circ}$ & 4.5 & 5.5 & 1.2 \\
90 & $2^{\circ}$ & 4.8 & 7.8 & 1.6 \\
180 & $1^{\circ}$ & 6.5 & 28.9 & 4.5 \\
360 & $30^{\prime}$ & 15.4 & 193.9 & 12.6 \\
720 & $15^{\prime}$ & 61.0 & 1518.4 & 24.0 \\
900 & $2^{\prime}$ & 100.3 & 3004.8 & 30.0 \\
1800 & $6^{\prime}$ & 574.2 & 24204.4 & 42.2 \\
3000 & $3^{\prime}$ & 751.2 & 37065.2 & 49.3 \\
\hline
\end{tabular}

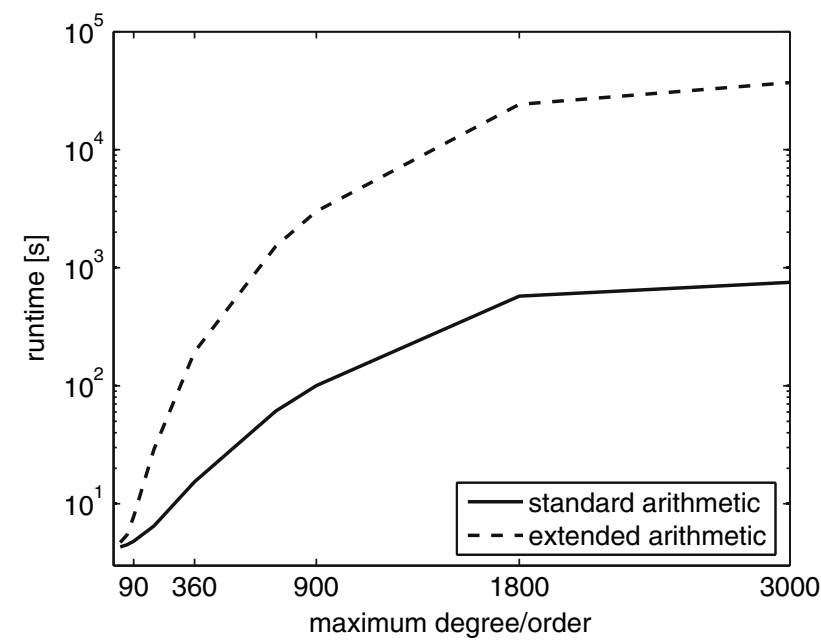

Fig. 4 SHS run-times for standard (solid line) and extended-range (dashed line) arithmetic as a function of maximum degree $n_{\max }$. Note the logarithmic scale

two reported in (Smith et al. 1981) for a single ALF. This is because the computation of the ALFs is not the only operation in SHA and SHS that has to be executed in extended-range arithmetic.

A similar test was done for the SHA programs SHA and ERA-SHA. As the standard arithmetic program SHA is considered to be stable only up to degree 720 , this was selected as $n_{\max }$. Results are shown in Table 2 and Fig. 5. The additional computation time needed for extended-range SHA is not as large as for SHS. For $n_{\max }=720$, about 12 times more computation time is needed. This is about half the value for SHS.

We tested the stability of the SHS programs for degrees up to 3, 000. We compared the results of GEOGFG18 by Wenzel (1998), HARMONIC SYNTH by Holmes and Pavlis, and our extended-range ERA-SHS. All tests were performed using a synthetic model complete to degree 3,000. The coefficients up to degree 2,160 were derived from EGM96 (Lemoine et al. 1998) and a global digital terrain model according to the procedure proposed by Claessens (2002). The model was extrapolated beyond degree 2,160 using Kaula's rule of thumb for degree variances.

The comparison is done for height anomalies, which were synthesized along a profile at $90^{\circ}$ longitude. GEOGFG18 is supposed to be stable up to degree 1,900 , while $H A R$ MONIC SYNTH is supposed to be stable up to degree 2, 700. This leads to the following four test setups and expected outcomes:

- Maximum degree and order $n_{\max }=1,800$. Profile from $-89^{\circ} 57.5^{\prime}$ to $89^{\circ} 57.5^{\prime}$ at $5^{\prime}$ spacing. All programs should work for this setup.

- Maximum degree and order $n_{\max }=2,160$. Profile from $-89^{\circ} 57.5^{\prime}$ to $89^{\circ} 57.5^{\prime}$ at $5^{\prime}$ 'spacing. GEOGFG18 should give erroneous results.

- Maximum degree and order $n_{\max }=2,700$. Profile from $-89^{\circ} 58.5^{\prime}$ to $89^{\circ} 58.5^{\prime}$ at $3^{\prime}$ spacing. HARMONIC SYNTH should still work correctly.

- Maximum degree and order $n_{\max }=3,000$. Profile from $-89^{\circ} 58.5^{\prime}$ to $89^{\circ} 58.5^{\prime}$ at $3^{\prime}$ spacing. This should give erroneous results with both GEOGFG18 and HARMONIC SYNTH.

The values computed by GEOGFG18 and HARMONIC $S Y N T H$ were compared to the values from ERA-SHS, which was used as a benchmark. For instance, for $n=100,000$ and a spherical grid with spacing $\frac{180^{\circ}}{n}$, only 10 decimal figures are lost when computing ALFs in extended-range arithmetic, according to Eq. (3).

The results for $n_{\max }=1,800$ are shown in Fig. 6. As expected, the height-anomaly errors are very small, with amplitudes $<1 \mathrm{~mm}$. Note that HARMONIC SYNTH produces slightly different results than GEOGFG18, which we attribute to different implementations. The extended-range arithmetic program $E R A-S H S$ delivers identical results at uncritical latitudes, which is expected because ERA-SHS is based on GEOGFG18. 
Table 2 SHA run-times and run-time ratios for standard and extended-range arithmetic at various maximum degrees $n_{\max }$

\begin{tabular}{llccr}
\hline$n_{\max }$ & Spacing & Standard $[\mathrm{s}]$ & Extended $[\mathrm{s}]$ & Ratio \\
\hline 30 & $5^{\circ}$ & 0.02 & 0.03 & 1.8 \\
60 & $2.5^{\circ}$ & 0.07 & 0.22 & 3.2 \\
90 & $1.5^{\circ}$ & 0.23 & 0.94 & 4.1 \\
180 & $48^{\prime}$ & 1.38 & 10.7 & 7.7 \\
360 & $24^{\prime}$ & 14.52 & 154.25 & 10.6 \\
540 & $18^{\prime}$ & 59.99 & 687.14 & 11.5 \\
720 & $12^{\prime}$ & 205.96 & 2403.1 & 11.7 \\
\hline
\end{tabular}

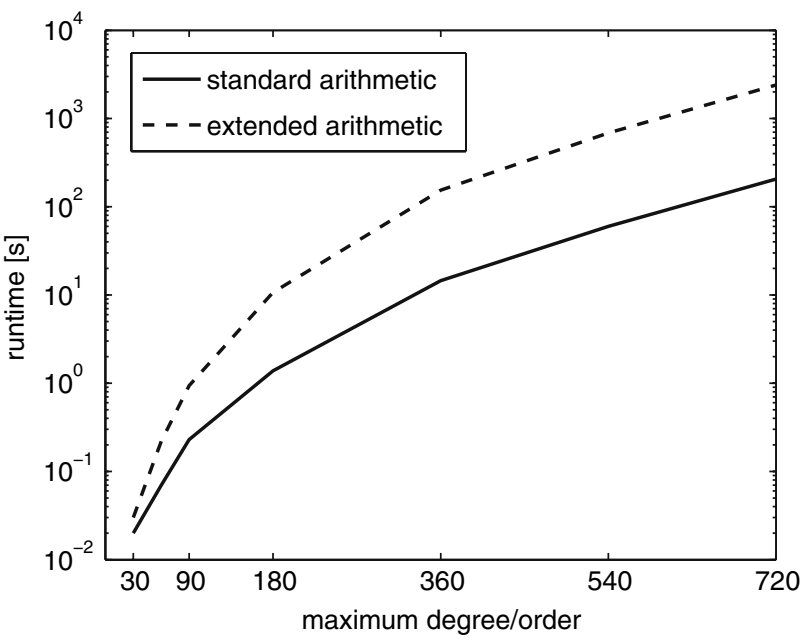

Fig. 5 SHA run-times for standard (solid line) and extended-range (dashed line) arithmetic. Logarithmic scale

Figure 7 shows the results for the second test case, $n_{\max }=2,160$. As expected, HARMONIC SYNTH still produces stable results with a maximum height-anomaly error of $0.3 \mathrm{~mm}$. GEOGFG18 works fine up to latitudes of approximately $\pm 50^{\circ}$, but errors increase beyond that, up to $1.9 \mathrm{~m}$.

The errors for $n_{\max }=2,700$ are shown in Fig. 8. The height-anomaly errors produced by GEOGFG18 increase, with a maximum error of $51.7 \mathrm{~m}$. However, good results are obtained for latitudes smaller than $\pm 45^{\circ}$. HARMONIC SYNTH produces good results over almost the whole latitude range. The errors increase towards the North Pole, reaching $1 \mathrm{~mm}$ at approximately $82^{\circ}$ latitude, with a maximum error of $4.1 \mathrm{~cm}$.

Finally, the height-anomaly errors for $n_{\max }=3,000$ are shown in Fig. 9. As expected, GEOGFG18 and HARMONIC SYNTH produce large errors. GEOGFG18 starts producing errors at latitudes just below $\pm 45^{\circ}$, the maximum error is $147.6 \mathrm{~m}$. The maximum error for HARMONIC SYNTH is $29.8 \mathrm{~m}$. Errors start exceeding $1 \mathrm{~mm}$ at latitudes of $\pm 60^{\circ}$.

The benefit of using extended-range arithmetic in SHA has been verified with a test computing spherical harmonic coefficients up to degree 3,000 . As input data, uniformly
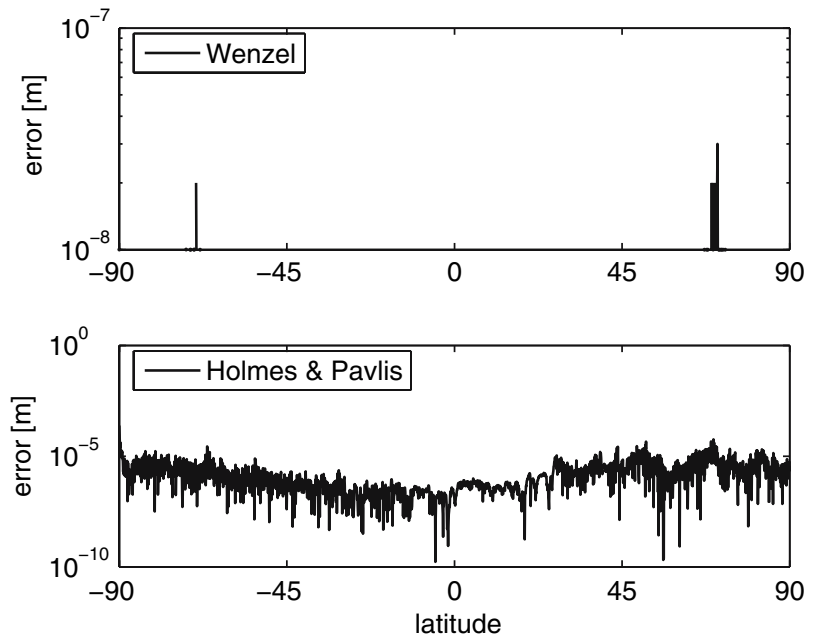

Fig. 6 SHS errors in terms of height anomalies (in metres) for $n_{\max }=1,800$. Top GEOGFG18, maximum error is $0.03 \mu \mathrm{m}$. Bottom HARMONIC SYNTH, maximum error is $0.2 \mathrm{~mm}$

distributed random numbers on a global grid with three-minute spacing were generated. This results in a "worse case" scenario as, unlike with real data, the power in the higher frequencies does not diminish. In real applications, the errors are expected to be smaller.

From this data set, potential coefficients were computed using (i) the routine $S H A$ and (ii) the routine ERA-SHA. The difference between the two is shown in Fig. 10. The difference is shown as

$\frac{\sum_{m}\left|\bar{c}_{l, m}-\bar{c}_{l, m}^{r e f}\right|^{2}}{\sum_{m}\left|\bar{c}_{l, m}^{r e f}\right|^{2}}$,

with the extended arithmetic solution serving as reference solution. Figure 10 shows very small differences up to about degree 1, 700. Beyond that, errors quickly increase to a point where the standard double precision solution is totally meaningless.

The test shows that, at least with this worst-case scenario, extended-range arithmetic is required if meaningful coefficients are to be computed beyond degree 1, 700 . 

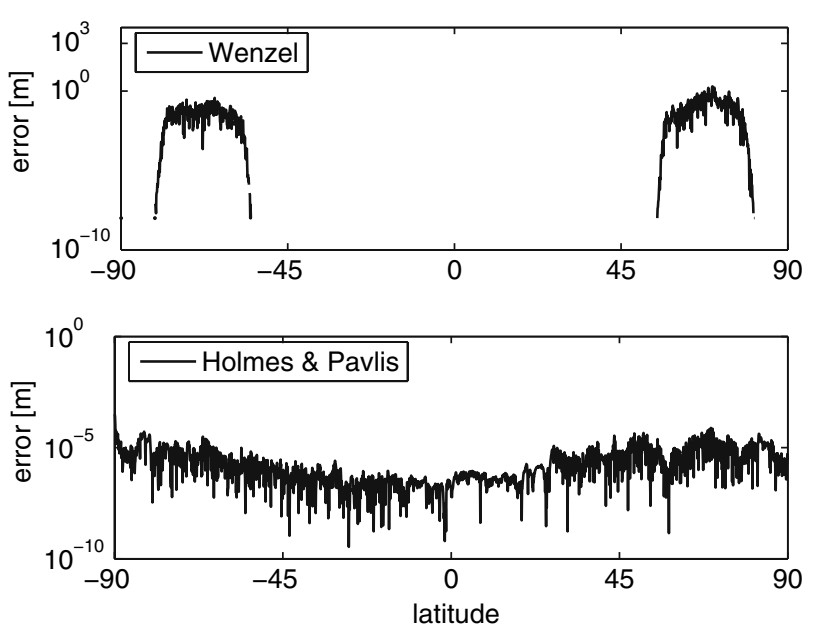

Fig. 7 SHS errors in terms of height anomalies (in metres) for $n_{\max }=$ 2, 160. Top GEOGFG18, maximum error is $1.9 \mathrm{~m}$. Bottom HARMONIC SYNTH, maximum error is $0.3 \mathrm{~mm}$
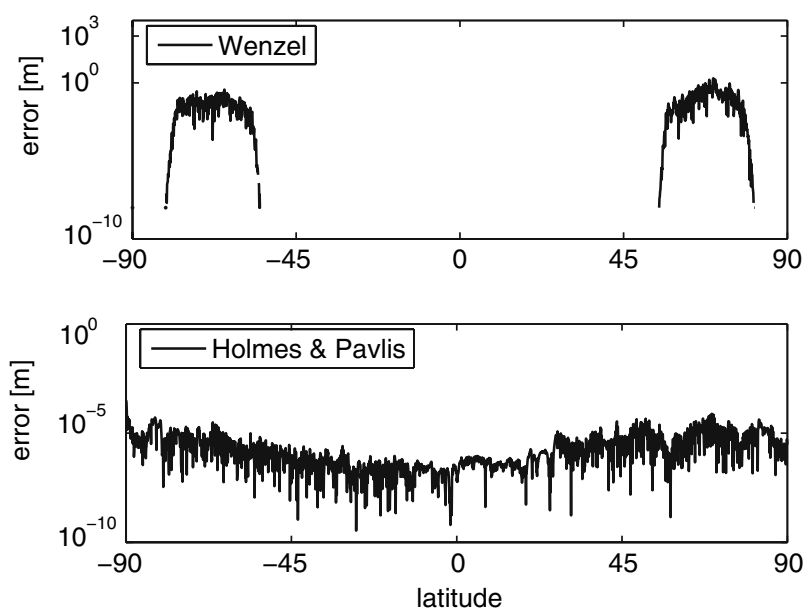

Fig. 8 SHS errors in terms of height anomalies (in metres) for $n_{\max }=2$, 700. Top GEOGFG18, maximum error is $51.7 \mathrm{~m}$. Bottom HARMONIC SYNTH, maximum error is $4.1 \mathrm{~cm}$

\section{Summary and conclusions}

We have developed routines for SHA and SHS, which use some open source extended-range software of the SLATEC Common Mathematical Library. The routines allow SHA and SHS up to essentially arbitrary degrees for all co-latitudes in the interval $\left(0^{\circ}, 180^{\circ}\right)$. The current implementation of the extended-range arithmetic is significantly more time-consuming than standard arithmetic, although there is scope for optimizing the computational efficiency. Hence, for the time being and for applications where time is a limiting factor, the approach by Jekeli et al. (2007) is preferred. However, for applications, where time is not a limiting factor, extendedrange arithmetic offers very accurate results and gives a high level of confidence that the results of scientific computations
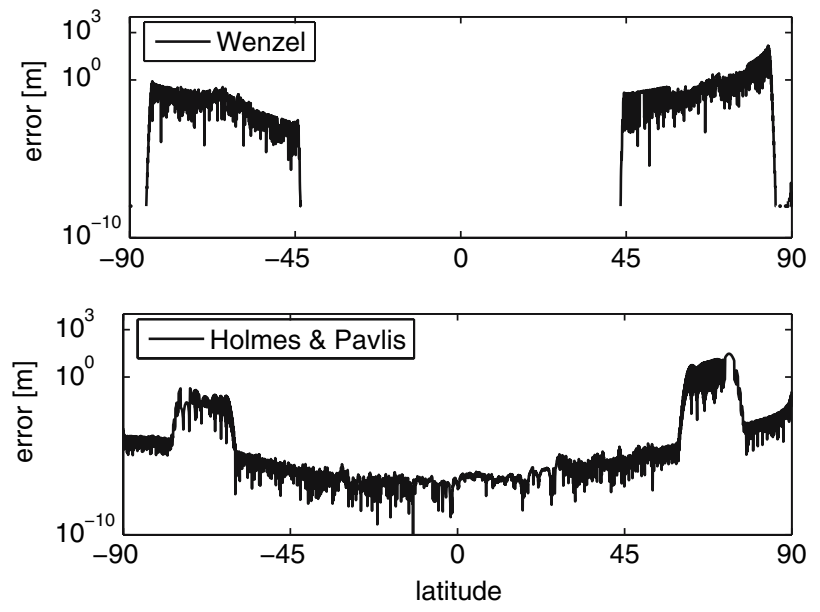

Fig. 9 SHS errors in terms of height anomalies (in metres) for $n_{\max }=$ 3, 000. Top GEOGFG18, maximum error is $147.6 \mathrm{~m}$. Bottom HARMONIC SYNTH, maximum error is $29.8 \mathrm{~m}$

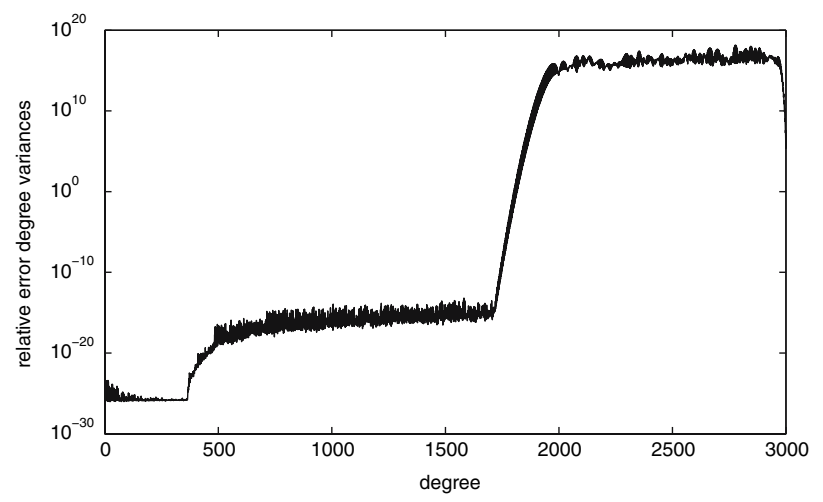

Fig. 10 Relative degree variances of the difference between the standard double precision SHA program SHA and the extended-range arithmetic SHA program $E R A-S H A$

are not corrupted by numerical instabilities, which may affect proper interpretation.

The extended-range SHA and SHS programs allow validation of existing SHA and SHS software for high-degree SHA and SHS. The tests of the publicly available routines GEOGFG18 and HARMONIC SYNTH confirm the expected accuracy and range of application of these routines. However, they also showed their limitations. Users of these routines have to take into account that errors increase quickly with decreasing distance to the North Pole, whereas the South Pole is less critical. Moreover, for equatorial areas and mid-latitudes, the admissible range of degrees of these routines may increase, beyond degree 1, 800 and 2,700, respectively. The source code of the programs ERA-SHA and ERA-SHS will be provided on request. Please contact T. Wittwer (t.f.wittwer@ tudelft.nl) or K. Seitz (seitz@ gik.uni-karlsruhe.de).

Acknowledgments The routine GEOGFG18 was made publicly available by H.G. Wenzel a long time ago. N.K. Pavlis provided us 
with the latest version of HARMONIC SYNTH; the program can be downloaded from http://earth-info.nima.mil/GandG/. This support is gratefully acknowledged. We also would like to thank three anonymous reviewers for their valuable comments.

\section{References}

Brendt RP (1978) A Fortran multiple-precision arithmetic package. ACM Trans Math Softw 4:57-70

Claessens SJ (2002) A synthetic earth model - Analysis, implementation, validation and application. MSc Thesis, Delft University of Technology, Physical and Space Geodesy group, Delft

Driscoll JR, Healy D (1994) Computing Fourier transforms and convolutions on the 2-sphere. Adv Appl Math 15:202-250

Freeden W, Windheuser U (1997) Combined spherical harmonics and wavelet expansion a future concept in earth's gravitational potential determination. Appl Comput Harmonic Anal 4:1-37

Gleason DM (1985) Partial sums of Legendre series via Clenshaw summation. Manuscr Geod 10:115-130

Heck B, Seitz K (1991) Harmonic analysis - spherical harmonicanalysis of gridded data. Technical Report, Geodetic Institute, University of Karlsruhe, Karlsruhe

Heck B, Seitz K (1993) Effects of non-linearity in the geodetic boundary value problems. Deutsche Geodätische Kommission (DGK) Reihe C, Heft Nr. 109, München, pp. 17-22

Holmes SA (2003) High degree spherical harmonic synthesis for simulated earth gravity modelling. PhD Thesis, Department of Spatial Sciences, Curtin University of Technology, Perth, Australia, $171 \mathrm{pp}$

Holmes SA, Featherstone WE (2002) A unified approach to the Clenshaw summation and the recursive computation of very high degree and order normalized associated Legendre functions. J Geod 76:279299

Jekeli C, Lee JK, Kwon JH (2007) On the computation and approximation of ultra-high-degree spherical harmonic series. J Geod. Doi: 10.1007/s00190-006-0123-Z
Koop R, Stelpstra D (1989) On the computation of the gravitational potential and its first and second derivatives. Manuscr Geod 14:373382

Lemoine FG, Kenyon SC, Factor JK, Trimmer RG, Pavlis NK, Chinn DS, Cox CM, Klosko SM, Luthcke SB, Torrence MH, Wang YM, Williamson RG, Pavlis EC, Rapp RH, Olson TR (1998) The development of the joint NASA GSFC and the National Imagery and Mapping Agency (NIMA) geopotential model EGM96, NASA/ TP-1998-206861, National Aeronautics and Space Administration, Greenbelt, 575 pp

Libbrecht KG (1985) Practical considerations for the generation of large order spherical harmonics. Solar Phys 99:371-373

Lozier DW, Smith JM (1981) Algorithm 567 Extended-range arithmetic and normalized Legendre polynomials. ACM Trans Math Softw 7:141-146

Olver FWJ, Smith JM (1983) Associated Legendre functions on the cut. J Comput Phys 51:502-518

Pavlis NK, Holmes SA, Kenyon SC, Schmidt D, Trimmer R (2005) A preliminary gravitational model to degree 2160 . In: Jekeli C, Bastos L, Fernandes J (eds) Gravity, geoid and space missions. International Association of Geodesy Symposia, vol 129. Springer, Berlin, pp.18-23

Smith JM, Olver FWJ, Lozier DW (1981) Extended-range arithmetic and normalized Legendre polynomials. ACM Tran Math Softw 7:93-105

Tscherning CC, Rapp RH, Goad C (1983) A comparison of methods for computing gravimetric quantities from high degree spherical harmonic expansions. Manuscr Geod 8:249-272

Tscherning CC, Poder K (1982) Some geodetic applications of Clenshaw summation. Boll Geod Sci Aff 4:351-364

Wenzel HG (1998) Ultra high degree geopotential models GPM98A and GPM98B to degree 1,800. In: Proceedings of joint meeting international gravity commission and international geoid commission, Budapest, 10-14 March. Rep 98:4, Finnish Geodetic Institute, Helsinki, pp 71-80 